\title{
Cardiovascular magnetic resonance findings in patients with PRKAG2 gene mutations
}

\author{
Pauli Pöyhönen ${ }^{1 *}$, Anita Hiippala ${ }^{2}$, Laura Ollila ${ }^{1}$, Touko Kaasalainen ${ }^{3,4}$, Helena Hänninen ${ }^{1}$, Tiina Heliö ${ }^{1}$, Jonna Tallila ${ }^{5}$,
} Catalina Vasilescu' ${ }^{6}$, Sari Kivistö ${ }^{3}$, Tiina Ojala ${ }^{2}$ and Miia Holmström ${ }^{3}$

\begin{abstract}
Background: Autosomal dominantly inherited PRKAG2 cardiac syndrome is due to a unique defect of the cardiac cell metabolism and has a distinctive histopathology with excess intracellular glycogen, and prognosis different from sarcomeric hypertrophic cardiomyopathy. We aimed to define the distinct characteristics of PRKAG2 using cardiovascular magnetic resonance (CMR).
\end{abstract}

Methods: CMR (1.5 T) and genetic testing were performed in two families harboring PRKAG2 mutations. On CMR, segmental analysis of left ventricular (LV) hypertrophy (LVH), function, native T1 mapping, and late gadolinium enhancement (LGE) were performed.

Results: Six individuals (median age 23 years, range 16-48; two females) had a PRKAG2 mutation: five with an R302Q mutation (family 1), and one with a novel H344P mutation (family 2). Three of six mutation carriers had LV mass above age and gender limits $(203 \mathrm{~g} / \mathrm{m} 2,157 \mathrm{~g} / \mathrm{m} 2$ and $68 \mathrm{~g} / \mathrm{m} 2)$ and others (with R302Q mutation) normal LV masses. All mutation carriers had LVH in at least one segment, with the median maximal wall thickness of 13 mm (range 11-37 mm). Two R302Q mutation carriers with markedly increased LV mass (203 g/m2 and $157 \mathrm{~g} / \mathrm{m} 2$ ) showed a diffuse pattern of hypertrophy but predominantly in the interventricular septum, while other mutation carriers exhibited a non-symmetric mid-infero-lateral pattern of hypertrophy. In family 1, the mutation negative male had a mean T1 value of 963 ms, three males with the R302Q mutation, LVH and no LGE a mean value of $918 \pm 11$ ms, and the oldest male with the R302Q mutation, extensive hypertrophy and LGE a mean value of $973 \mathrm{~ms}$. Of six mutations carriers, two with advanced disease had LGE with 11 and $22 \%$ enhancement of total LV volume.

Conclusions: PRKAG2 cardiac syndrome may present with eccentric distribution of $L V H$, involving focal mid-infero-lateral pattern in the early disease stage, and more diffuse pattern but focusing on interventricular septum in advanced cases. In patients at earlier stages of disease, without LGE, T1 values may be reduced, while in the advanced disease stage T1 mapping may result in higher values caused by fibrosis. CMR is a valuable tool in detecting diffuse and focal myocardial abnormalities in PRKAG2 cardiomyopathy.

\section{Background}

PRKAG2 cardiac syndrome is an autosomal dominantly inherited metabolic heart muscle disease characterized by left ventricular hypertrophy $(\mathrm{LVH})$, progressive conducting abnormalities and ventricular pre-excitation (Wolff-Parkinson-White [WPW] syndrome) [1-4]. The prevalence is approximately $0.23-1 \%$ in patients with hypertrophic cardiomyopathy (HCM) $[4,5]$. Despite of the rarity, PRKAG2 accounts for a greater proportion of

\footnotetext{
* Correspondence: pauli.poyhonen@helsinki.fi

${ }^{1}$ Heart and Lung Center, University of Helsinki and Helsinki University Hospital, Po BOX 340, Helsinki 00029 HUCH, Finland

Full list of author information is available at the end of the article
}

$\mathrm{HCM}$ in children and adolescents [6], and patients may benefit from early identification due to high risk of complete atrioventricular block [4, 7] and sudden cardiac death caused by atrial fibrillation and rapid antegrade conduction through an accessory pathway [8].

Patients with a PRKAG2 gene defect have a distinctive cardiac histopathology with excess intracellular vacuoles filled with glycogen, myocyte enlargement but without myocyte disarray typical for sarcomeric HCM [3]. However, there are relatively few histopathological reports on PRKAG2 syndrome hearts, and in some cases abundant interstitial fibrosis and myocyte disarray in the absence of glycogen accumulation has been reported [4, 9]. Recently, 
severe fibrofatty myocardial replacement has been described in a patient with end-stage PRKAG2 [10].

In HCM, standard cardiovascular magnetic resonance (CMR) with late gadolinium enhancement (LGE) technique enables accurate assessment of myocardial morphology and function. While LGE visualizes the expansion of extracellular space related to focal myocardial fibrosis or infiltration [11, 12], T1 mapping has become a promising tool for evaluation of diffuse myocardial disease. Native T1 mapping reflects both intra- and extracellular signal in the myocardium [13]. Elevated native T1 times have been reported in fibrosis [14], edema [15] and amyloidosis [16], and lowered in iron overload [17] and focal fat infiltration [18]. Recently, native T1 mapping showed potential in the identification of Anderson-Fabry disease, a genetic storage disease characterized by multiorgan involvement and sphingolipid accumulation within myocytes [18, 19]. Metabolic defect resulting in macromolecular intracellular glycogen, and even lipid accumulation, may reduce native T1-relaxation time $[20,21]$, in the absence of significant extracellular fibrosis which is often seen in other forms of HCM.

To our knowledge, two case reports of CMR findings in PRKAG2 cardiomyopathy have been reported [22, 23]. As PRKAG2 cardiac syndrome is a unique glycogen storage cardiomyopathy, we sought whether CMR might demonstrate different imaging characteristics from sarcomeric HCM or hypertensive heart disease.

\section{Methods}

\section{Patient population}

This study was performed at the Helsinki University Central Hospital. All participants gave written informed consent and the project was approved by the local institutional ethics committee. Seven subjects from two separate families harboring PRKAG2 mutations were recruited to this study. Two of the patients had a pacemaker system [24].

Clinical evaluation of all study subjects comprised review of hospital records, 12-lead electrocardiography (ECG) and echocardiography (index patients). Patients underwent electrophysiological study based on the treating cardiologist's decision.

The phenotypic triad of PRKAG2 cardiac syndrome was based on observed cardiac hypertrophy, pre-excitation and conduction system disease [25]. Ventricular preexcitation was defined as a short PR interval $(<120 \mathrm{~ms})$, widened QRS interval $(>110 \mathrm{~ms})$, and an abnormal initial QRS vector (delta-wave), or demonstration of an antegradely conducting accessory pathway on electrophysiologic study. Evidence of supraventricular tachycardia in association with pre-excitation defined WPW [25]. Diagnosis of conduction system disease required sinus node dysfunction or atrioventricular block on ECG.

\section{Genetic analysis}

A novel next generation sequencing (NGS) strategy, oligonucleotide-selective sequencing [26], targeting 69 known genes associated with cardiomyopathies (Core Cardiomyopathy Panel, Blueprint Genetics, Helsinki, Finland, http://blueprintgenetics.com/), was used to examine the index patient in family 1 . Median sequence coverage per base pair was $137 \mathrm{x}$ and $97.3 \%$ of the target region was covered with $>15 x$. Sequence analysis identified a heterozygous missense variant in the PRKAG2 gene, c.905G > A (p.R302Q), which has been well characterized by several scientific reports as a disease mutation associated with dominantly inherited WPW and severe LVH resembling clinically $\operatorname{HCM}[2,7,9]$. Direct Sanger sequencing was used to confirm the detected variant and family member testing.

The index patient in family 2 was screened using a custom-designed panel of 117 cardiomyopathy-related genes (HaloPlex kit of 500Kb, Agilent Technologies) and a heterozygous missense mutation was identified in the PRKAG2 gene. The novel mutation p.H344P $(\mathrm{c} .1031 \mathrm{~A}>\mathrm{C})$ was shown to segregate with the disease in the family and was absent in 3250 Finnish controls (Sequencing Initiative Suomi (SISu) database, http://www.sisuproject.fi/). The p.H344P mutation alters an evolutionarily conserved site in the protein, indicating that this amino acid presents an important role for the structure and the function of the protein. The variant was rated as deleterious and possibly damaging by the bioinformatics prediction tools SIFT and PolyPhen-2 respectively, strengthening the idea that this is a pathogenic variant.

\section{CMR technique}

CMR was performed with 1.5 T MR scanner (Avantofit; Siemens, Erlangen, Germany) using a 32-channel receiver cardiac coil. Breath-hold cine MR was performed using retrospectively electrocardiographically gated segmented true fast imaging with balanced steady-state free precession (bSSFP) TrueFISP sequence. To assess left (LV) and right (RV) ventricular volumes and ejection fractions (EF) cine MR images were obtained in vertical, and horizontal longaxis, and a stack of short-axis planes covering both ventricles. Three-chamber view was obtained to assess LV outflow tract. The typical imaging parameters were TR/TE 3.0/1.6 ms, flip angle $52^{\circ}, 256 \times 256$ matrix, $240 \times 340 \mathrm{~mm}$ field of view (FOV). Slice thickness was $6 \mathrm{~mm}$ and interslice gap $20 \%$. The temporal resolution was $42-49 \mathrm{~ms}$.

Myocardial T1 mapping was performed in a midventricular short-axis slice using a shortened Modified Look-Locker Inversion-recovery (ShMOLLI) sequence. Typical acquisition parameters for ShMOLLI sequence were TR/TE $2.1 / 1.1 \mathrm{~ms}$, flip angle $35^{\circ}, 236 \times 256$ matrix and $331 \times 360 \mathrm{~mm}$ FOV, inversion times varying from $90 \mathrm{~ms}$ to circa $5000 \mathrm{~ms}$, and $8 \mathrm{~mm}$ slice thickness. 
Quantitative myocardial T2 mapping was performed in a breath-hold fashion by using a T2-prepared bSSFP sequence to produce three single-shot T2-weighted images, each with different $\mathrm{T} 2$-preparation times $\left(\mathrm{TE}_{\mathrm{T} 2 \mathrm{P}}=0 \mathrm{~ms}\right.$, $25 \mathrm{~ms}$ and $55 \mathrm{~ms}$ ). The other scanning parameters were as follows: $\mathrm{TR}=4 \times \mathrm{RR}$ intervals, flip angle $35^{\circ}, 192 \times 154$ matrix, $360 \times 289 \mathrm{~mm}$ FOV, and $8 \mathrm{~mm}$ slice thickness.

Ten minutes after injection of a contrast agent (gadoteratemeglumine, Dotarem ${ }^{\circledR} 0.2 \mathrm{mmol} / \mathrm{kg}$ ) LGE images were acquired in the same views as for cine images, using inversion recovery spoiled gradient echo (IR-SPGR) sequence. The imaging parameters were TR/TE 2.58/2.3 ms, flip angle $50^{\circ}, 256 \times 256$ matrix, $240 \times 340$ FOV. Slice thickness was $8 \mathrm{~mm}$ and interslice gap $0 \%$. Inversion time was optimized for each measurement to null the signal intensity of normal myocardium (240-360 ms).

Imaging of two patients with a permanent pacemaker device were performed according to previously published MR safety protocol for the pacemaker patients [24].

\section{Image analysis}

Images were analyzed in consensus with two experienced (>10 years of experience) cardiac radiologists (SK, $\mathrm{MH})$. In each patient, the three LV short axis sections were divided into 17 segments according to American Heart Association (AHA) guidelines [27]. Segments with any artifacts due to a pacemaker were excluded in all analysis.

Volumes, mass and wall thickness were assessed using standard protocols [28]. Imaging analysis was performed using QMass MR software ${ }^{\bullet}$ (version 7.6, Medis Medical Imaging Systems, Leiden, Netherlands). Cardiac hypertrophy was defined as increased LV wall thickness in one or more myocardial segments, or RV free wall thickness, of more than two standard deviations above the population mean (z-score $>2$ ) on CMR.

Motion corrected T1 maps were generated and T1 estimates were computed on a per-pixel basis by performing a non-linear curve fitting using the three parameter signal model. We performed "midwall myocardial" segmental T1 analysis by measuring the mean T1-relaxation values separately for the anterior, anteroseptal, inferoseptal, inferior, inferolateral and anterolateral segments of myocardium (six segments). In addition to segmental analysis we also calculated the mean T1 value of the mid-myocardium. T1 values were compared with published values for normal healthy myocardium [29].

Acquired T2 images were motion corrected to inplane motion between images by using a fast non-rigid registration algorithm. A pixel-wise myocardial T2 maps were generated using curve-fitting based on two parameter equation by assuming mono-exponential signal decay. Segmental analysis was performed for myocardial T2 values similarly as for $\mathrm{T} 1$ values.
The LGE percentage of LV was calculated using QMass MR software ${ }^{\circ}$. Hyperenhanced pixels were defined using full width at half maximum method.

\section{Statistical analysis}

Continuous variables are presented as median (range) or mean \pm standard deviation (SD) as appropriate, and categorical variables as frequency (\%), unless otherwise mentioned. Comparison between continuous normally distributed variables was performed with Student's $t$-test.

Results are given as absolute values and standardized to age, gender, body surface area (BSA, Mosteller's method) and normal myocardial function (z-scores). Normal ventricular volumes and masses for adults were obtained from the references [28, 30] and for children ( $<18$ years) from the reference [31], using similar $1.5 \mathrm{~T}$ MR scanner and bSSFP pulse sequence as ours.

Mean and maximal wall thickness of each of the $16 \mathrm{LV}$ segments (apex left out) are presented as absolute ( $\mathrm{mm}$ ) and standardized (z-scores) values, with the aim to estimate the real distribution of segmental hypertrophy in relation to normal changes in wall thickness through the myocardium. Normal LV wall thickness values for adults were obtained from the reference [32] (similar scanner, sequence and QMass software tool as ours). Maximal absolute and standardized wall thickness values do not always represent the same segment.

Currently, there are no available reference values for LV wall thickness based on the 17-segment-model for children. Therefore, we used adult CMR references for the 17-year-old female (BSA $1.83 \mathrm{~m} 2$ ) and for the 16-yearold male (BSA $1.33 \mathrm{~m} 2$ ). BSA-standardized echocardiographic reference values were also used for all children for measurement of maximal wall thickness [33].

To take into account clustering and correlation of data within patients and myocardial segments, the association between segmental T1 values and hypertrophy were analysed using a linear mixed effects model, with patients treated as a random intercept and segments having a fixed effect on $\mathrm{T} 1$.

A $p$-value of $<0.05$ was considered statistically significant and all tests were 2-sided. Statistical analysis was performed using the SPSS 21 package (SPSS, Chigaco, IL, USA). R language and environment for statistical computing (Version 2.15.3, R Core Team 2013, R Foundation for Statistical Computing, Vienna, Austria) was used for graphical output of segmental LV wall thickness and linear mixed effects model analysis.

\section{Results}

Study patients

Of the seven individuals examined with CMR in two separate families (Fig. 1a and b), altogether six had a PRKAG2 mutation: five R302Q mutations in family 1 

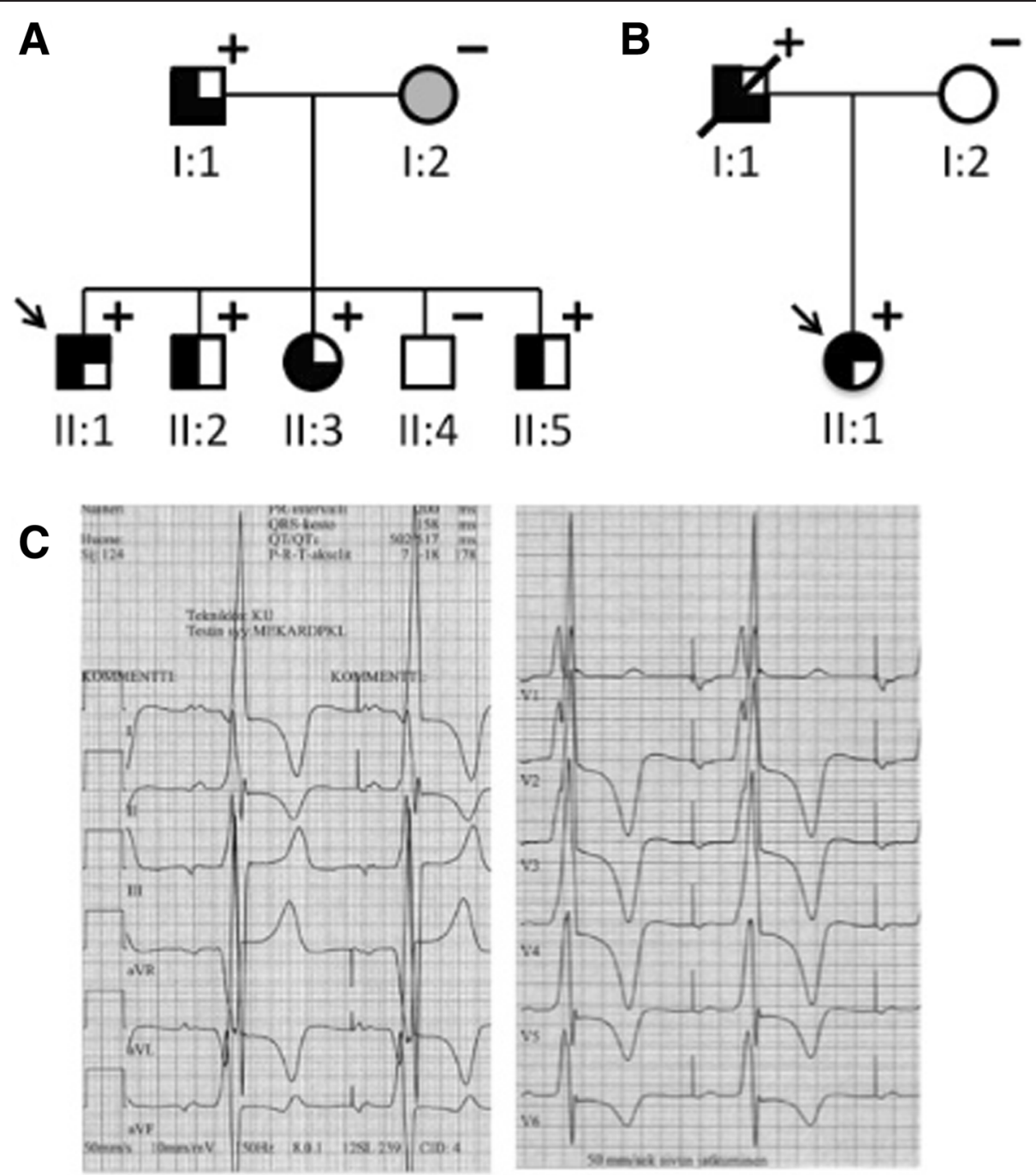

Fig. 1 Pedigrees of two families with PRKAG2 cardiac syndrome and consequent clinical phenotypes of patients. Individuals with a PRKAG2 mutation (+) were identified, with R302Q substitution in family 1 (a) and H344P substitution in family 2 (b). Squares indicate males and circles females. Filled symbols indicate disease phenotype in affected individuals, i.e., cardiac hypertrophy (left half filled), pre-excitation (right upper quadrant filled), or conduction system disease (right lower quadrant filled). Open symbols denote unaffected individuals and shading denotes uncertain clinical status. Arrows indicate index patients. The electrocardiogram of a patient with the R302Q mutation (c). Atrial pacing with large QRS deflections and pathologic T waves

and one novel H344P mutation in family 2. The median age of the six mutation carriers (two females) was 23 years (range 16-48 years) and BSA $1.76 \mathrm{~m}^{2}$ (range 1.33$1.88 \mathrm{~m}^{2}$ ). One 19-year-old male did not have a PRKAG2 mutation. Six mutation carriers completed otherwise full CMR imaging protocol, but one patient missed $\mathrm{T} 1$ and $\mathrm{T} 2$ mapping.

Clinical features of examined family members are presented in Table 1, and an abnormal electrocardiogram of a patient with the R302Q mutation in Fig. 1c. Based on hospital records, no other risk factors for LVH such as hypertension or chronic kidney diseases were detected in patients examined with CMR.

\section{Ventricular volumes, function and hypertrophy}

All six PRKAG2 mutation carriers had preserved age, gender and BSA standardized LV and RV end-diastolic volume (EDV), stroke volume and EF, see Table 2. However, three of six had LV mass above age and gender limits. In family 1, two R302Q mutation carriers (aged 23 and 48 years) had markedly elevated LV mass $(203 \mathrm{~g} / \mathrm{m} 2$ [z-score 18.8] and $157 \mathrm{~g} / \mathrm{m} 2$ [9.6]) (Fig. 2) and three (aged 16, 24 and 26 years) normal LV masses (Fig. 3). In family 2, the 17-year-old female with an H344P mutation had also elevated LV mass (68 g/m2 [2.2]).

All mutation carriers had LVH by definition, i.e., wall thickness $>2$ z-scores in one or more LV myocardial 
Table 1 Clinical features of study individuals

\begin{tabular}{|c|c|c|c|c|c|c|c|c|c|c|c|c|c|c|c|c|c|}
\hline $\begin{array}{l}\text { PRKAG2 } \\
\text { mutation }\end{array}$ & $\begin{array}{l}\text { Age at } \\
\text { CMR (yrs) }\end{array}$ & $\begin{array}{l}\text { Age at } \\
\text { Dx (yrs) }\end{array}$ & Sex & Pre-excitation & WPW & $\begin{array}{l}\text { Conduction } \\
\text { system } \\
\text { disease }^{a}\end{array}$ & PM & $\begin{array}{l}\text { EP study } \pm \\
\text { RF ablation }\end{array}$ & Syncope & $\begin{array}{l}\text { Cardiac } \\
\text { hypertrophy }\end{array}$ & $T x$ & Death & $\begin{array}{l}\text { Blood pressure } \\
\text { (systolic/diastolic) } \\
\mathrm{mmHg}\end{array}$ & $\begin{array}{l}\text { NYHA } \\
\text { functional } \\
\text { class }\end{array}$ & $\begin{array}{l}\text { Serum } \\
\text { Creatinine } \\
(\mu \mathrm{mol} / \mathrm{l})\end{array}$ & $\begin{array}{l}\text { Hemoglobin } \\
(\mathrm{g} / \mathrm{l})\end{array}$ & $\begin{array}{l}\text { NT-proBNB } \\
\text { (ng/l) }\end{array}$ \\
\hline \multicolumn{18}{|l|}{1} \\
\hline R302Q & 48 & 30 & M & - & - & + & + & - & + & + & - & - & $136 / 80$ & 1 & 91 & 165 & b \\
\hline R302Q & 26 & 8 & M & + & + & - & - & + & + & + & - & - & $113 / 69$ & 1 & 83 & 153 & 31 \\
\hline R302Q & 24 & 24 & M & - & - & - & - & + & - & + & - & - & $110 / 57$ & 1 & b & b & 25 \\
\hline R302Q & 23 & 5 & $\mathrm{~F}$ & - & - & + & + & - & - & + & - & - & $127 / 56$ & 1 & 63 & 115 & 1078 \\
\hline R302Q & 16 & 16 & M & - & - & - & - & - & - & + & - & - & $115 / 63$ & 1 & b & b & b \\
\hline \multicolumn{18}{|l|}{ y 2} \\
\hline H344P & c & 24 & M & - & - & + & + & - & + & + & + & + & $160 / 100$ & 3 & 58 & 120 & 39 \\
\hline H344P & 17 & 10 & $\mathrm{~F}$ & + & + & - & - & + & - & + & - & - & $116 / 65$ & 1 & 129 & 146 & 1330 \\
\hline
\end{tabular}

Mutation nomenclature is based on GenBank accession NM_016203.3 (PRKAG2) with nucleotide one being the first nucleotide of the translation initiation codon ATG

Abbreviations: CMR cardiovascular magnetic resonance, $D x$ diagnosis, EP electrophysiologic, $F$ female, $L V H$ left ventricular hypertrophy, $M$ male, NT-proBNB N-terminal of the prohormone brain natriuretic peptide, NYHA New York Heart Association, PM permanent pacemaker, RF radiofrequency, Tx cardiac transplantation, WPW Wolff-Parkinson-White syndrome

a Sinus node dysfunction or atrioventricular block on ECG

aSinus node dysfunction
bData not available

'Was not examined with CMR. Cardiac hypertrophy diagnosis was based on the explanted heart 
Table 2 Ventricular volumes, function and masses of PRKAG2 mutation carriers compared to age, gender and body surface area standardized reference values

\begin{tabular}{|c|c|c|}
\hline & All & \\
\hline & $(n=6)$ & \\
\hline & Value & $z$-score ${ }^{a}$ \\
\hline Left ventricle & & \\
\hline End-diastolic volume, $\mathrm{ml}$ & $163(116-180)$ & \\
\hline End-diastolic volume index, $\mathrm{ml} / \mathrm{m} 2$ & $90(82-100)$ & $0.6(-0.1-1.6)$ \\
\hline End-systolic volume, $\mathrm{ml}$ & $63(38-72)$ & \\
\hline End-systolic volume index, ml/m2 & $34(28-42)$ & $0.9(-1.0-2.2)$ \\
\hline Stroke volume, ml & $99(78-109)$ & \\
\hline Stroke volume index, $\mathrm{ml} / \mathrm{m} 2$ & $57(52-59)$ & $0.3(-0.3-1.0)$ \\
\hline Cardiac output, I/min & $5.9(4.8-8.2)$ & \\
\hline Cardiac output index, $1 /\left(\min ^{\mathrm{a}} \mathrm{m} 2\right)$ & $3.5(3.1-4.4)$ & \\
\hline LVEF, \% & $62(58-67)$ & $-0.3(-1.5-1.5)$ \\
\hline Mass, $\mathrm{g}$ & $137(96-335)$ & \\
\hline Mass index, $\mathrm{g} / \mathrm{m} 2$ & $78(68-203)$ & $2.1(-0.1-18.8)$ \\
\hline Right ventricle & & \\
\hline End-diastolic volume, $\mathrm{ml}$ & $167(124-189)$ & \\
\hline End-diastolic volume index, $\mathrm{ml} / \mathrm{m} 2$ & $95(75-102)$ & $0.3(-1.0-0.9)$ \\
\hline End-systolic volume, $\mathrm{ml}$ & $68(38-83)$ & \\
\hline End-systolic volume index, ml/m2 & $39(23-45)$ & $0.2(-1.3-1.3)$ \\
\hline Stroke volume, $\mathrm{ml}$ & $99(78-106)$ & \\
\hline Stroke volume index, $\mathrm{ml} / \mathrm{m} 2$ & $56(52-59)$ & $0.2(-0.2-0.7)$ \\
\hline Cardiac output, $1 /$ min & $5.8(4.9-8.2)$ & \\
\hline Cardiac output index, $1 /\left(\min ^{\mathrm{a}} \mathrm{m} 2\right)$ & $3.5(3.1-4.4)$ & \\
\hline RVEF, \% & $59(56-69)$ & $0.1(-1.1-1.3)$ \\
\hline
\end{tabular}

Values are median (range)

Abbreviations: $L V E F$ left ventricular ejection fraction, RVEF right ventricular ejection fraction

a Z-scores were calculated using the normal reference values for adults by Maceira A. et al. [30] and children by Buechel E. et al. [31]

segments, with the median maximal wall thickness of $13 \mathrm{~mm}$ (range 11-37 $\mathrm{mm}$ ) and z-score of 3.2 (range 2.3-19.0), see Table 3. Segmental distribution of maximal wall thickness had two different patterns (Fig. 4). Two symptomatic R302Q mutation carriers (aged 48 and 23 years) with markedly increased LV mass had hypertrophy throughout the myocardium, but predominantly in the interventricular septum (Fig. 4a and d), with the maximal (based on z-scores) wall thickness of $31 \mathrm{~mm}$ (z-score 11.8) and $37 \mathrm{~mm}$ (19.0) in the mid-anteroseptal segment in both patients. Trabeculae or papillary muscles were excluded in the measurements. Other mutation carriers with normal or only mildly increased LV mass (three R302Q carriers: aged 26, 24 and 16 years; one H344P carrier: aged 17 years) had LV hypertrophy in a non-symmetric pattern with the maximal wall thickness located in mid-inferolateral or mid-inferior segments (Fig. 4b, c, e and f).

The median RV free wall thickness was $5 \mathrm{~mm}$ (range 2-6 $\mathrm{mm}$ ).

\section{T1 and T2 mapping}

In family 1 , the mutation negative male (19 years old) had a mean T1 value of $963 \mathrm{~ms}$, see Table 4. Three male siblings (26, 24 and 16 years) with the R302Q mutation, asymmetric LVH and without LGE, had a mean T1 value of $918 \pm 11 \mathrm{~ms}$. The oldest male in the family (48 years) with the R302Q mutation, extensive hypertrophy and LGE, had a mean T1 value of $973 \mathrm{~ms}$ (mid-anteroseptal, -anterior and -anterolateral segments excluded due to artifact caused by pacemaker), while corresponding segments had a mean LGE volume of $8 \%$.

Considering 18 non-enhanced mid-myocardial segments in three male R302Q carriers, there was a trend toward significant inverse association between segmental T1 times and hypertrophy (segments with wall thickness z-score $>2$ had a mean T1 value of $909 \pm 16$ ms versus other segments $925 \pm 17 \mathrm{~ms} ; p=0.054)$. However, after adjustment to fixed effects caused by different segments on T1, the association between segmental T1 times and hypertrophy was nonsignificant ( $p=0.194$; linear mixed effects model).

In family 2, the female H344P mutation carrier (17 years) with asymmetric LVH and without LGE, had a mean midmyocardium T1 value of 966 ms.

T2-relaxation times were within normal limits in all patients and there were no significant associations between hypertrophied segments and T2 time.

\section{LGE}

Of the six mutations carriers, only two had left ventricular LGE, with $11 \%$ and $22 \%$ enhancement of total LV volume (Fig. 2). These patients also had severe LV hypertrophy. LGE was patchy and diffuse but focusing on the most hypertrophic segments. None of the patients presented LGE in the right ventricle.

\section{Histology}

The father in family 2 (H344P carrier), who did not undergo CMR, had diffuse cardiac hypertrophy, LV and RV wall thickness ad 31 and $9 \mathrm{~mm}$, intracellular vacuolization with positive periodic acid-Schiff (PAS) staining indicative of glycogen, and focal fibrosis in the explanted heart. There was also increased amount of left and right atrial fat.

\section{Mutation negative sibling}

The 19-year-old mutation negative sibling in family 1 had normal LV mass and wall thickness, normal RV free wall thickness, but increased LV EDV (111 ml/m2 [2.8]) and decreased EF $55 \%$ [-2.2]. 


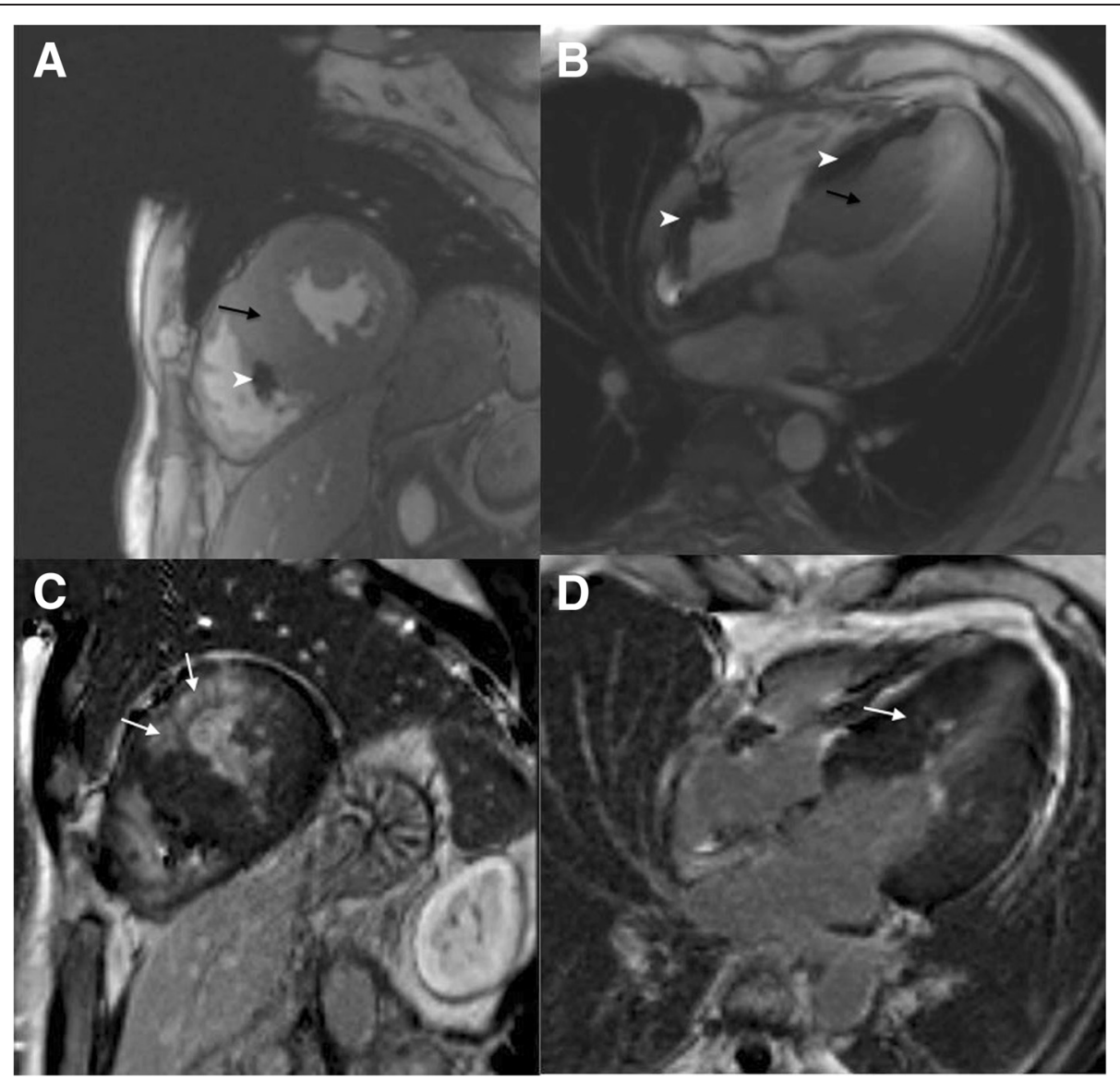

Fig. 2 A 48-year-old male patient with PRKAG2 cardiac syndrome and a pacemaker. In short-axis and four chamber cine images septum is severely hypertrophied (a-b) (black arrows). Maximal septal and lateral wall thickness was $31 \mathrm{~mm}$ and $25 \mathrm{~mm}$, respectively. Papillary muscles were excluded in the measurements. Anteroseptal, hypertrophic areas exhibit patchy late gadolinium enhancement (c-d) (white arrows). White arrow heads indicate artefact from pacemaker lead

\section{Discussion}

We are the first to describe the comprehensive CMR findings of six individuals with PRKAG2 mutation, known to cause a unique defect of the cardiac cell metabolism with intracellular deposition of glycogen. We found that the distribution of LVH in PRKAG2 syndrome is eccentric. Patients with markedly increased LV mass showed a diffuse pattern of hypertrophy but predominantly in the interventricular septum. On the other hand, four patients with normal or only mildly increased LV mass exhibited a non-symmetric mid-infero-lateral pattern of hypertrophy. $\mathrm{T} 1$ values were lowest in patients with the R302Q mutation, LVH and no LGE. Two patients with the PRKAG2 mutation and advanced disease showed intramyocardial, patchy LGE in hypertrophic segments.

\section{Hypertrophy}

We found that in five patients with the R302Q mutation (family 1), LVH demonstrated a similar diffuse pattern in two patients with markedly increased LV mass, but a nonsymmetric mid-infero-lateral pattern in three patients with normal LV masses. Also, one patient with the H344P mutation (family 2 ) presented with non-symmetric midinfero-lateral pattern of LVH. Significant LVH in PRKAG2 patients have been demonstrated previously by echocardiography [7, 34]. In the largest series of 45 PRKAG2 mutation carriers, $78 \%$ had LVH on echocardiography with a mean LV wall thickness of $21 \mathrm{~mm}$ (range 13-45 mm), and varying pattern of hypertrophy within families, $46 \%$ having concentric, $29 \%$ asymmetric, and one distal hypertrophy, with often eccentric distributions [4]. While concentric hypertrophy seems to be more common in metabolic and infiltrative cardiomyopathies [6], in sarcomeric HCM the distribution of $\mathrm{LVH}$ is characteristically asymmetric and heterogeneous, preferentially involving basal interventricular septum and often extending into the lateral wall, the posterior septum and LV apex [35].

Our finding of both diffuse and non-symmetric distribution of LVH in the same family may be explained by the generally progressive nature of LVH in PRKAG2 cardiomyopathy during follow-up [4], and the varying age of disease onset. That is, PRKAG2 mutations may express in 


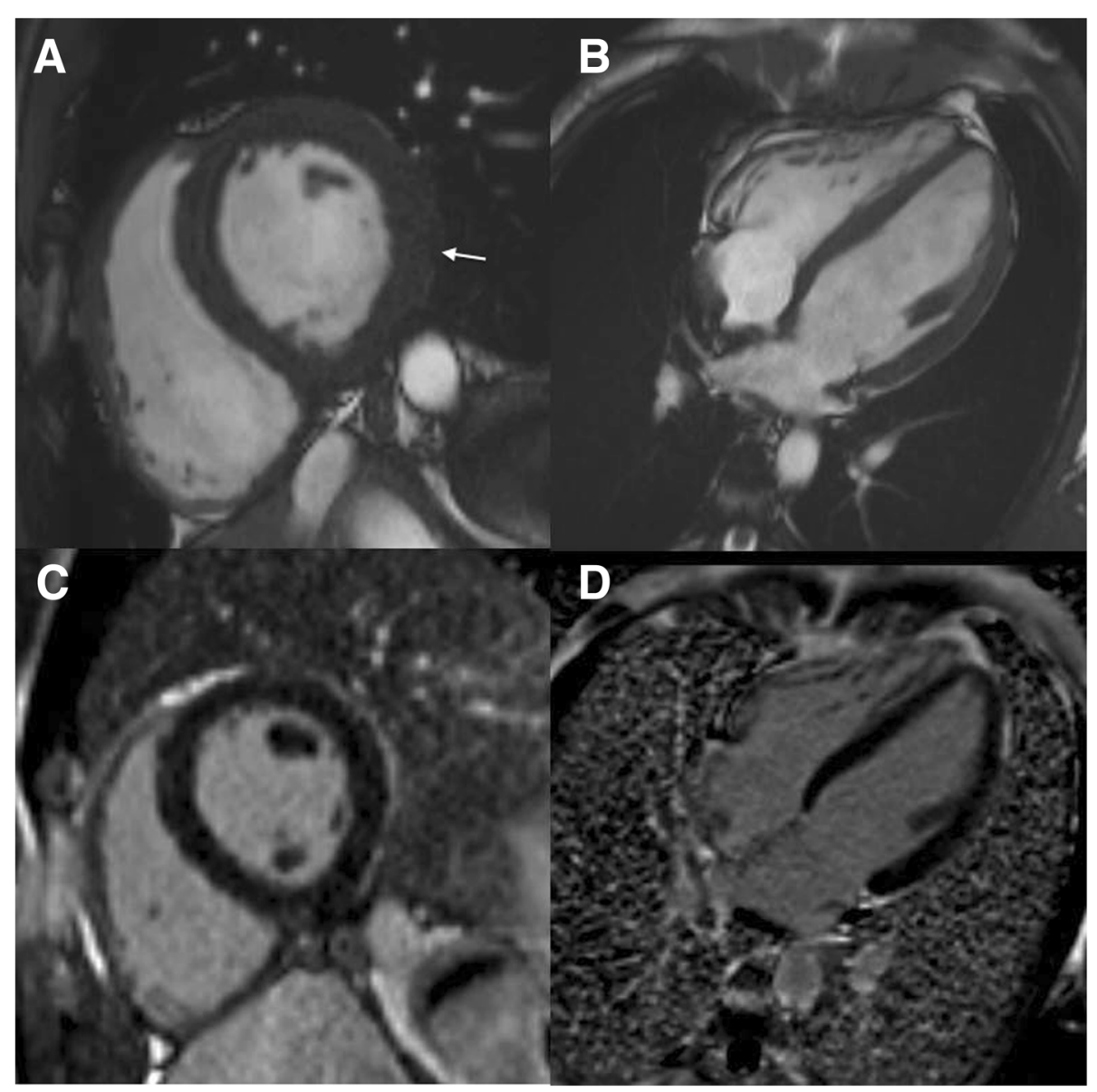

Fig. 3 A 16-year-old male patient with a PRKAG2 mutation. In short-axis cine image (a-b) inferolateral left ventricular wall is mildly hypertrophied (10-11 mm, maximal z-score 2.3) (white arrow), no late gadolinium enhancement is present (c-d)

Table 3 Summary of ventricular wall thickness compared to normal reference values and late gadolinium enhancement of PRKAG2 mutation carriers

\begin{tabular}{|c|c|c|}
\hline & \multicolumn{2}{|l|}{ All } \\
\hline & \multicolumn{2}{|l|}{$\overline{(n=6)}$} \\
\hline & Value & $z$-score ${ }^{a}$ \\
\hline \multicolumn{3}{|l|}{ Left ventricle } \\
\hline Left ventricular hypertrophy ${ }^{\mathrm{b}}$ & $6(100)$ & \\
\hline Maximal wall thickness, mm & $13(11-37)$ & $3.2(2.3-19.0)$ \\
\hline LGE presence & $2(33)$ & \\
\hline LGE extent, \% left ventricular volume ${ }^{c}$ & $17(11-22)$ & \\
\hline \multicolumn{3}{|l|}{ Right ventricle } \\
\hline Free wall thickness, mm & $5(2-6)$ & \\
\hline LGE presence & $0(0)$ & \\
\hline
\end{tabular}

Values are median (range) or $\mathrm{n}(\%)$

Abbreviation: $L G E$ late gadolinium enhancement

${ }^{a}$ Standardized wall thickness (z-score), see Statistics section

beft ventricular hypertrophy was defined as $>2 z$-score wall thickening in one or more myocardial segments

'Only two patients had LGE the early stage with focal and mild LVH, which may change to diffuse disease in the later stage. Another explanation could be, that even in the same family and with the same mutation, there would be varying patterns of hypertrophy. Nevertheless, our study demonstrates that CMR based accurate segmental analysis of wall thickness revealed abnormal hypertrophy in all PRKAG2 mutation carriers, even in those subjects with normal LV mass.

\section{T1 and T2 mapping}

Our study is the first one describing the behaviour of $\mathrm{T} 1$ or $\mathrm{T} 2$ times in a glycogen storage cardiomyopathy. In family 1 , three males with the R302Q mutation, LVH but no LGE, presented with lower mean mid-myocardial T1 values (918 $\pm 11 \mathrm{~ms})$ compared to mutation negative male sibling $(963 \mathrm{~ms})$ of their age and the mutation positive male with extensive hypertrophy and LGE (973 ms). This is in accordance with the hypothesized $\mathrm{T} 1$ reduction by intracellular glycogen in the absence of significant fibrosis. Theoretically, intracellular glycogen as a macromolecule with hydrophilic bonding with water would 

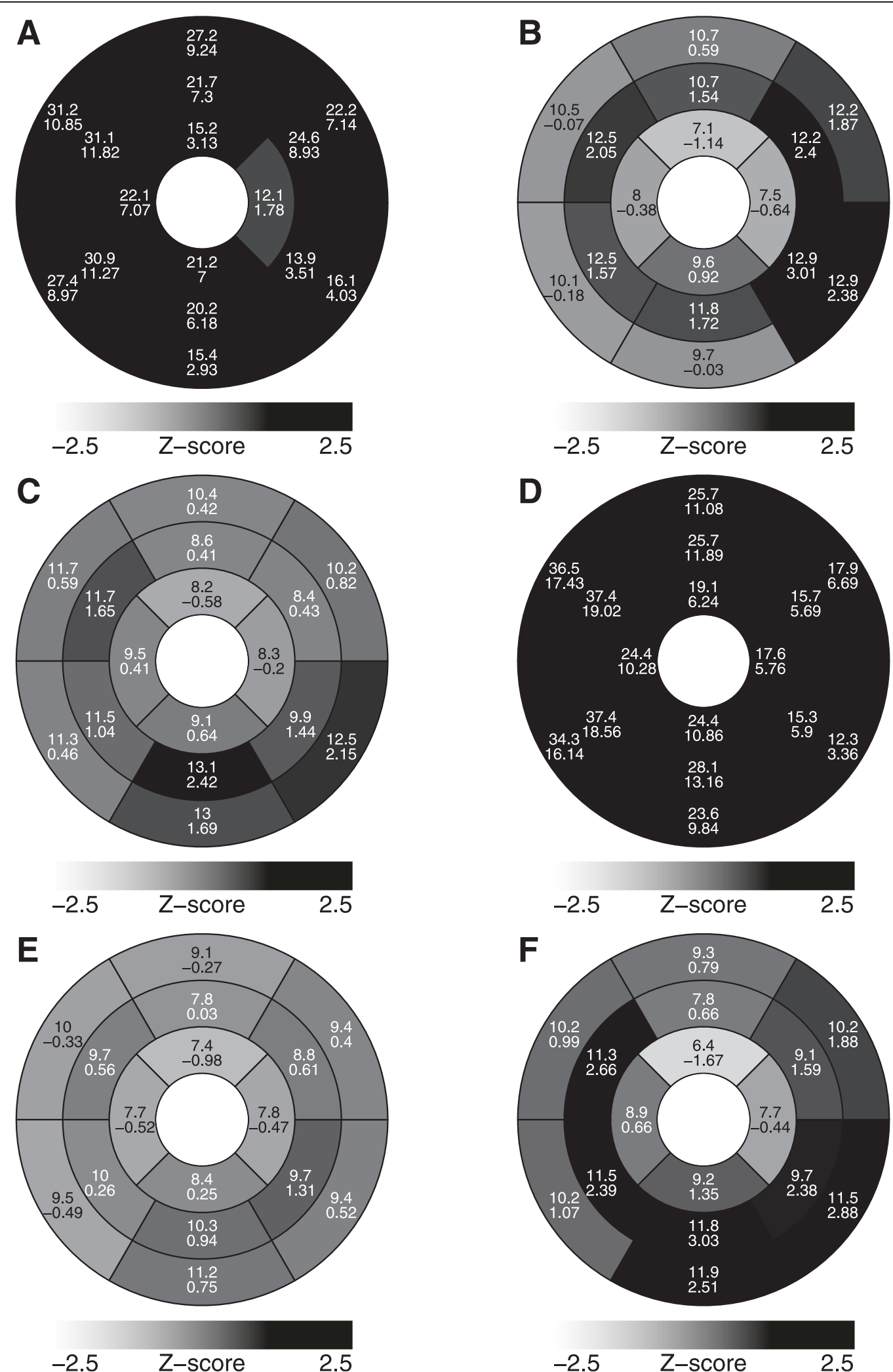

Fig. 4 (See legend on next page.)

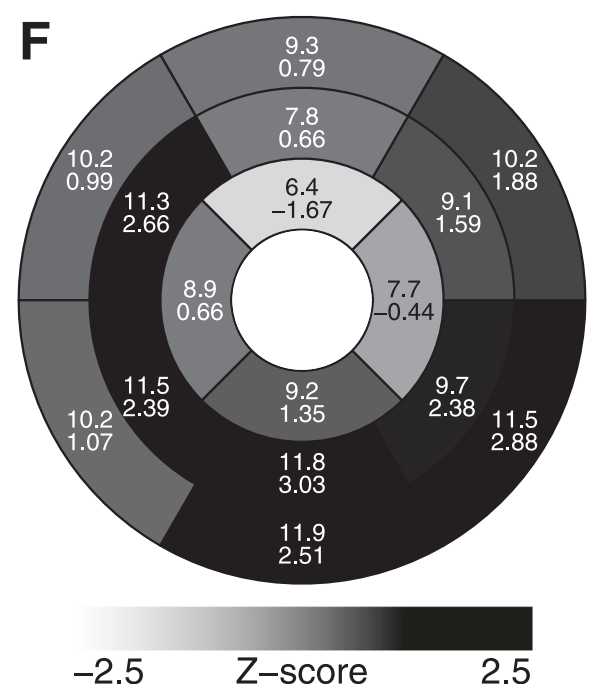


(See figure on previous page.)

Fig. 4 Distribution of left ventricular hypertrophy of six PRKAG2 mutation carriers. Upper and lower values represent absolute (mm) and standardized (z-score) maximal wall thickness in the segment using adult references. Gray scaling of each segment is based on z-score. Members of family 1 with an R302Q mutation: I:1 (a), II:1 (b), II:2 (c), II:3 (d) and Il:4 (e). A member of family 2 with an H344P-mutation: II:1 (f). The 16-year-old male (e), with body-surface-area (BSA) of $1.33 \mathrm{~m} 2$, had hypertrophy in mid-infero-lateral segment (posterior free wall) of $10 \mathrm{~mm}$ ( $\mathrm{z}$-score 2.3 ) using the BSA-standardized echocardiography based reference values for children

reduce T1-relaxation time, as shown in vitro [20, 21]. Possible lipid accumulation, demonstrated in a case report of end-stage PRKAG2, might also potent T1 shortening [10]. On the other hand, in the advanced disease stage T1 values may be slightly increased as a result of pseudonormalization caused by extracellular fibrosis, as seen in Anderson-Fabry disease $[18,19]$.

We were unable to verify the hypothesis of inverse association between segmental T1 times and hypertrophy in non-enhanced myocardium. After adjustment to fixed effects caused by different segments on T1, the association between T1 times and hypertrophy was non-significant. This may be due to limited number of patients and the fact that LVH was predominantly located in mid-inferolateral segments which also present lower T1 values in normal myocardium [36].

In a previous study, it was estimated that normal native myocardial ShMOLLI T1 time is $962 \pm 25 \mathrm{~ms}$ [29]. Piechnik et al. also showed that the principal biological parameter influencing myocardial ShMOLLI T1 is the female gender, $\mathrm{T} 1$ times of females under 45 years being $24 \mathrm{~ms}$ longer (blood $130 \mathrm{~ms}$ ). In above 45-year-olds ShMOLLI T1 values did not differ between the sexes. The same study also reported no age-dependency of either myocardial or blood $\mathrm{T} 1$ values in males. In our very limited study population, the mean myocardial $\mathrm{T} 1$ value

Table 4 Mid-myocardium native T1 and T2 values of six study individuals (one R302Q mutation carrier lacked T1 and T2 mapping data)

\begin{tabular}{|c|c|c|c|c|c|c|c|}
\hline & \multicolumn{6}{|l|}{ Segment } & \multirow[b]{2}{*}{ Mean } \\
\hline & Anterior & Anteroseptal & Inferoseptal & Inferior & Inferolateral & Anterolateral & \\
\hline \multicolumn{8}{|l|}{ Native T1 values } \\
\hline \multicolumn{8}{|l|}{ Family $1(n=5)$} \\
\hline \multicolumn{8}{|l|}{ R302Q mutation $(n=4)$} \\
\hline \multicolumn{8}{|l|}{ LGE negative $(n=3)$} \\
\hline 1 & 934 & 932 & 944 & 905 & 897 & 929 & 924 \\
\hline 2 & 914 & 902 & 925 & 924 & 879 & 894 & 906 \\
\hline 3 & 922 & 938 & 945 & 919 & 905 & 918 & 925 \\
\hline Mean & 923 & 924 & 938 & 916 & 894 & 914 & 918 \\
\hline LGE positive $(n=1)$ & m. v. & m. v. & 990 & 944 & 986 & m. v. & 973 \\
\hline No mutation $(n=1)$ & 984 & 954 & 938 & 960 & 984 & 955 & 963 \\
\hline \multicolumn{8}{|l|}{ Family $2(n=1)$} \\
\hline H344P mutation $(n=1)$ & 950 & 965 & 985 & 968 & 957 & 969 & 966 \\
\hline \multicolumn{8}{|l|}{ Native $T 2$ values } \\
\hline \multicolumn{8}{|l|}{ Family $1(n=5)$} \\
\hline \multicolumn{8}{|l|}{ R302Q mutation $(n=4)$} \\
\hline \multicolumn{8}{|l|}{ LGE negative $(n=3)$} \\
\hline 1 & 42.8 & 44.4 & 39.9 & 39.7 & 43.9 & 40.5 & 41.9 \\
\hline 2 & 41.4 & 44.3 & 43.8 & 43.0 & 43.2 & 40.3 & 42.7 \\
\hline 3 & 42.1 & 46.5 & 42.2 & 40.5 & 45.5 & 40.2 & 42.8 \\
\hline Mean & 42.1 & 45.1 & 42.0 & 41.1 & 44.2 & 40.3 & 42.5 \\
\hline LGE positive $(n=1)$ & m. v. & m. v. & 41.9 & 40.2 & 39.9 & m. v. & 40.7 \\
\hline No mutation $(n=1)$ & 43.3 & 47.6 & 41.5 & 37.2 & 43.2 & 39.0 & 42.0 \\
\hline \multicolumn{8}{|l|}{ Family $2(n=1)$} \\
\hline H344P mutation $(n=1)$ & 50.4 & 50.0 & 49.0 & 45.4 & 50.4 & 45.4 & 48.4 \\
\hline
\end{tabular}


of the 17-year-old female H344P mutation carrier was 966 ms, whereas T1 values of male R302Q mutation carriers were from 906 to $973 \mathrm{~ms}$; thus the differences caused by gender and mutation type may attenuate the possible changes related to intracellular glycogen.

All our patients had normal T2-relaxation times. Normal T2 times exclude possible iron accumulation in the myocardium and its effect on T1 values [37, 38].

\section{Late gadolinium enhancement}

In this study, patients with significant LVH and increased LV mass, had widespread LGE. In an earlier case report of a patient with large cardiomyocyte vacuoles due to extensive cytosolic accumulation of glycogen in Danon disease (a mutation of the LAMP2 gene), CMR demonstrated both RV and LV dysfunction, and extensive homogeneous LGE of the RV with concomitant patchy midwall LV involvement [39]. This was suggested to be caused by sarcolemmal damage in an advanced storage disease and subsequent gadolinium retention within intracellular space [39]. Similar mechanism of LGE has been suggested in Anderson-Fabry disease, with intracellular lipid accumulation [18], and could also explain LGE in our patients with the progressive stage of PRKAG2.

\section{Study limitations}

The reference values used for calculating z-scores of LV wall thickness according to AHA 17-segment model were based on the largest available study on participants free of cardiac disease (similar software tool, MR scanner and sequence) with a mean age of $66 \pm 9$ years, which is considerably higher than our patients (median age 23 years, range 16-48). Therefore, we presented both absolute and standardized values of wall thickness when appropriate. In 16 myocardial segments, there is considerable probability to achieve abnormal z-scores in some segments by change. To overcome this, we plotted the distribution of $\mathrm{z}$-scores in each patient to study the consistency of values in adjacent segments.

Our cohort was too small to find correlations between age, symptom duration and disease severity. We did not study the time-related changes in the myocardium. However, according to an earlier study, cardiac hypertrophy has a generally progressive nature in PRKAG2 [4], and thus different patterns of LVH in the same family may represent different stages of the disease. This study also lacks histological validation of imaging findings, although we presented the histology of the explanted heart of the father in family 2.

\section{Conclusions}

PRKAG2 cardiac syndrome may present with eccentric distribution of $\mathrm{LVH}$, involving focal mid-infero-lateral pattern in the early disease stage, and more diffuse pattern but focusing on interventricular septum in advanced cases. In patients at earlier stages of the disease, without LGE, T1 values may be reduced, while in the advanced disease stage T1 mapping may result in higher values caused by fibrosis. CMR is a valuable tool in detecting diffuse and focal myocardial abnormalities in PRKAG2 cardiomyopathy.

\begin{abstract}
Abbreviations
BSA: Body surface area; bSSFP: Balanced steady-state free precession; CMR: Cardiovascular magnetic resonance; ECG: Electrocardiography; EDV: End-diastolic volume; EF: Ejection fraction; FOV: Field of view; HCM: Hypertrophic cardiomyopathy; IR-SPGR: Inversion recovery spoiled gradient echo; LGE: Late gadolinium enhancement; LV: Left ventricular; LVH: Left ventricular hypertrophy; NGS: Next generation sequencing; PAS: Periodic acid-Schiff; RV: Right ventricular; ShMOLLI: Shortened Modified Look-Locker Inversion-recovery; WPW: Wolff-Parkinson-White.
\end{abstract}

\section{Competing interests}

The authors declare that they have no competing interests.

\section{Authors' contributions}

PP participated in analysis and interpretation of data, statistical analysis and writing the manuscript. AH has done clinical evaluations of patients, taken part in patient and DNA collections and has been involved in drafting and revising the manuscript. LO has done phenotyping and clinical evaluation of patients and manuscript writing. TK was performing $\mathrm{T} 1$ and $\mathrm{T} 2$ measurements for the study, and participated also in writing and revising process of the manuscript. $\mathrm{HH}$ has made contributions to conception and design of the study and revising the manuscript. TH contributed to funding, phenotyping and evaluation of clinical data, writing. JT and CV have participated in analyzing DNA samples. SK has been involved in analyzing CMR images, drafting and revising the manuscript. TO has taken part in patient and DNA collection. MH has participated in planning the study protocol, organizing patient's CMR imaging, analyzing CMR images and writing the manuscript. All authors read and approved the manuscript.

\section{Acknowledgements}

We would like to acknowledge all the patients who participated in this study and research nurse Sini Weckström for assistance. PP received a grant from Ida Montin Foundation, TH received grants from the Finnish Foundation for Cardiovascular Research, Finnish Medical Foundation, Aarno Koskelo Foundation, and the special governmental subsidy (2014208) for health sciences research of the University Hospital of Helsinki. SK received a grant from the special governmental subsidy (YLD8114001) for health sciences research of the University Hospital of Helsinki.

\section{Author details}

${ }^{1}$ Heart and Lung Center, University of Helsinki and Helsinki University Hospital, Po BOX 340, Helsinki 00029 HUCH, Finland. ${ }^{2}$ Children's Hospital, University of Helsinki and Helsinki University Hospital, Helsinki, Finland. ${ }^{3} \mathrm{HUS}$ Medical Imaging Center, Radiology, University of Helsinki and Helsinki University Hospital, Helsinki, Finland. ${ }^{4}$ HUS Medical Imaging Center, Clinical Physiology and Nuclear Medicine, University of Helsinki and Helsinki University Hospital, Helsinki, Finland. ${ }^{5}$ Blueprint Genetics, Helsinki, Finland. ${ }^{6}$ Molecular Neurology Research Program, Biomedicum Helsinki, University of Helsinki, Helsinki, Finland.

Received: 17 August 2015 Accepted: 6 October 2015 Published online: 24 October 2015

\section{References}

1. MacRae CA, Ghaisas N, Kass S, Donnelly S, Basson CT, Watkins HC, et al. Familial Hypertrophic cardiomyopathy with Wolff-Parkinson-White syndrome maps to a locus on chromosome 7q3. J Clin Invest. 1995:96(3):1216-20.

2. Gollob MH, Green MS, Tang AS, Gollob T, Karibe A, Ali Hassan AS, et al. Identification of a gene responsible for familial Wolff-Parkinson-White syndrome. N Engl J Med. 2001;344(24):1823-31.

3. Arad M, Benson DW, Perez-Atayde AR, McKenna WJ, Sparks EA, Kanter RJ, et al. Constitutively active AMP kinase mutations cause glycogen storage 
disease mimicking hypertrophic cardiomyopathy. J Clin Invest 2002;109(3):357-62.

4. Murphy RT, Mogensen J, McGarry K, Bahl A, Evans A, Osman E, et al. Adenosine monophosphate-activated protein kinase disease mimicks hypertrophic cardiomyopathy and Wolff-Parkinson-White syndrome: natural history. J Am Coll Cardiol. 2005;45(6):922-30.

5. Gruner C, Care M, Siminovitch K, Moravsky G, Wigle ED, Woo A, et al Sarcomere protein gene mutations in patients with apical hypertrophic cardiomyopathy. Circ Cardiovasc Genet. 2011;4(3):288-95.

6. Authors/Task Force members, Elliott PM, Anastasakis A, Borger MA, Borggrefe M, Cecchi F, et al. 2014 ESC Guidelines on diagnosis and management of hypertrophic cardiomyopathy: the Task Force for the Diagnosis and Management of Hypertrophic Cardiomyopathy of the European Society of Cardiology (ESC). Eur Heart J. 2014;35(39):2733-79.

7. Sternick EB, Oliva A, Gerken LM, Magalhaes L, Scarpelli R, Correia FS, et al. Clinical, electrocardiographic, and electrophysiologic characteristics of patients with a fasciculoventricular pathway: the role of PRKAG2 mutation. Heart Rhythm. 2011;8(1):58-64.

8. Zhang LP, Hui B, Gao BR. High risk of sudden death associated with a PRKAG2-related familial Wolff-Parkinson-White syndrome. J Electrocardiol. 2011:44(4):483-6.

9. Tan $H L$, van der Wal AC, Campian ME, Kruyswijk HH, ten Hove JB, van Doorn DJ, et al. Nodoventricular accessory pathways in PRKAG2-dependent familial preexcitation syndrome reveal a disorder in cardiac development. Circ Arrhythm Electrophysiol. 2008;1(4):276-81.

10. Roberts JD, Veinot JP, Rutberg J, Gollob MH. Inherited cardiomyopathies mimicking arrhythmogenic right ventricular cardiomyopathy. Cardiovasc Pathol. 2010;19(5):316-20.

11. Desai MY, Ommen SR, McKenna WJ, Lever HM, Elliott PM. Imaging phenotype versus genotype in hypertrophic cardiomyopathy. Circ Cardiovasc Imaging. 2011;4(2):156-68.

12. Maron MS. Clinical utility of cardiovascular magnetic resonance in hypertrophic cardiomyopathy. J Cardiovasc Magn Reson. 2012;14:13-429X-14-13.

13. White SK, Sado DM, Flett AS, Moon JC. Characterising the myocardial interstitial space: the clinical relevance of non-invasive imaging. Heart. 2012;98(10):773-9.

14. Dass S, Suttie JJ, Piechnik SK, Ferreira VM, Holloway CJ, Banerjee R, et al. Myocardial tissue characterization using magnetic resonance noncontrast t1 mapping in hypertrophic and dilated cardiomyopathy. Circ Cardiovasc Imaging. 2012;5(6):726-33.

15. Ferreira VM, Piechnik SK, Dall'Armellina E, Karamitsos TD, Francis JM, Ntusi N, et al. T(1) mapping for the diagnosis of acute myocarditis using CMR: comparison to T2-weighted and late gadolinium enhanced imaging. JACC Cardiovasc Imaging. 2013;6(10):1048-58.

16. Karamitsos TD, Piechnik SK, Banypersad SM, Fontana M, Ntusi NB, Ferreira VM, et al. Noncontrast T1 mapping for the diagnosis of cardiac amyloidosis. JACC Cardiovasc Imaging. 2013;6(4):488-97.

17. Feng Y, He T, Carpenter JP, Jabbour A, Alam MH, Gatehouse PD, et al. In vivo comparison of myocardial T1 with $\mathrm{T} 2$ and $\mathrm{T} 2{ }^{*}$ in thalassaemia major. J Magn Reson Imaging. 2013;38(3):588-93.

18. Sado DM, White SK, Piechnik SK, Banypersad SM, Treibel T, Captur G, et al. Identification and assessment of Anderson-Fabry disease by cardiovascular magnetic resonance noncontrast myocardial T1 mapping. Circ Cardiovasc Imaging. 2013;6(3):392-8.

19. Pica S, Sado DM, Maestrini V, Fontana M, White SK, Treibel T, et al. Reproducibility of native myocardial T1 mapping in the assessment of Fabry disease and its role in early detection of cardiac involvement by cardiovascular magnetic resonance. J Cardiovasc Magn Reson. 2014;16:99-014-0099-4.

20. Ragozzino E. Proton spin-lattice relaxation in solutions of glycogen in water. Mol Phys. 1966;10:497-8.

21. Mitchell DG, Burk Jr DL, Vinitski S, Rifkin MD. The biophysical basis of tissue contrast in extracranial MR imaging. AJR Am J Roentgenol. 1987;149(4):831-7.

22. Fabris E, Brun F, Porto AG, Losurdo P, Vitali Serdoz L, Zecchin M, et al. Cardiac hypertrophy, accessory pathway, and conduction system disease in an adolescent: the PRKAG2 cardiac syndrome. J Am Coll Cardiol. 2013;62(9):e17.

23. Sternick EB, de Almeida AS, Rocha C, Gollob M. Myocardial infarction in a teenager. Eur Heart J. 2014;35(23):1558.
24. Kaasalainen T, Pakarinen S, Kivisto S, Holmstrom M, Hanninen H, Peltonen J, et al. MRI with cardiac pacing devices - safety in clinical practice. Eur J Radiol. 2014;83(8):1387-95.

25. Gollob MH, Green MS, Tang AS, Roberts R. PRKAG2 cardiac syndrome: familial ventricular preexcitation, conduction system disease, and cardiac hypertrophy. Curr Opin Cardiol. 2002;17(3):229-34.

26. Myllykangas S, Buenrostro JD, Natsoulis G, Bell JM, Ji HP. Efficient targeted resequencing of human germline and cancer genomes by oligonucleotideselective sequencing. Nat Biotechnol. 2011;29(11):1024-7.

27. Cerqueira MD, Weissman NJ, Dilsizian V, Jacobs AK, Kaul S, Laskey WK, et al. Standardized myocardial segmentation and nomenclature for tomographic imaging of the heart. A statement for healthcare professionals from the Cardiac Imaging Committee of the Council on Clinical Cardiology of the American Heart Association. Int J Cardiovasc Imaging. 2002;18(1):539-42.

28. Maceira AM, Prasad SK, Khan M, Pennell DJ. Normalized left ventricular systolic and diastolic function by steady state free precession cardiovascular magnetic resonance. J Cardiovasc Magn Reson. 2006:8(3):417-26.

29. Piechnik S, Ferreira V, Lewandowski A, Ntusi N, Banerjee R, Holloway C, et al. Normal variation of magnetic resonance $\mathrm{T1}$ relaxation times in the human population at 1.5 T using ShMOLLI. J Cardiovasc Magn Reson. 2013;15(1):13.

30. Maceira AM, Prasad SK, Khan M, Pennell DJ. Reference right ventricular systolic and diastolic function normalized to age, gender and body surface area from steady-state free precession cardiovascular magnetic resonance. Eur Heart J. 2006;27(23):2879-88.

31. Buechel E, Kaiser T, Jackson C, Schmitz A, Kellenberger C. Normal right- and left ventricular volumes and myocardial mass in children measured by steady state free precession cardiovascular magnetic resonance. J Cardiovasc Magn Reson. 2009;11(1):19.

32. Kawel N, Turkbey EB, Carr JJ, Eng J, Gomes AS, Hundley WG, et al. Normal left ventricular myocardial thickness for middle-aged and older subjects with steady-state free precession cardiac magnetic resonance: the multiethnic study of atherosclerosis. Circ Cardiovasc Imaging. 2012;5(4):500-8.

33. Pettersen MD, Du W, Skeens ME, Humes RA. Regression equations for calculation of z scores of cardiac structures in a large cohort of healthy infants, children, and adolescents: an echocardiographic study. J Am Soc Echocardiogr. 2008;21(8):922-34.

34. Blair E, Redwood C, Ashrafian H, Oliveira M, Broxholme J, Kerr B, et al. Mutations in the gamma(2) subunit of AMP-activated protein kinase cause familial hypertrophic cardiomyopathy: evidence for the central role of energy compromise in disease pathogenesis. Hum Mol Genet. 2001;10(11):1215-20.

35. Klues HG, Schiffers A, Maron BJ. Phenotypic spectrum and patterns of left ventricular hypertrophy in hypertrophic cardiomyopathy: morphologic observations and significance as assessed by two-dimensional echocardiography in 600 patients. J Am Coll Cardiol. 1995;26(7):1699-708.

36. Dabir D, Child N, Kalra A, Rogers T, Gebker R, Jabbour A, et al. Reference values for healthy human myocardium using a T1 mapping methodology: results from the International T1 Multicenter cardiovascular magnetic resonance study. J Cardiovasc Magn Reson. 2014;16(1):69.

37. Wood JC. Magnetic resonance imaging measurement of iron overload. Curr Opin Hematol. 2007;14(3):183-90.

38. Carpenter JP, He T, Kirk P, Roughton M, Anderson LJ, de Noronha SV, et al. Calibration of myocardial T2 and T1 against iron concentration. J Cardiovasc Magn Reson. 2014;16:62-014-0062-4.

39. Carbone I, Francone M, Chimenti C, Galea N, Russo M, Frustaci A. Images in cardiovascular medicine: Right ventricular late enhancement as a magnetic resonance marker of glycogen storage disease. Circulation. 2010;122(2):189-90. 